\title{
Estrogen and progesterone-receptor-positive stroma as a non-tumorous proliferation in kidneys: a possible metaplastic response to obstruction
}

\author{
Satish K Tickoo ${ }^{1}$, Anuradha Gopalan ${ }^{1}$, Jiangling J Tu², Lara R Harik ${ }^{1}$, \\ Hikmat A Al-Ahmadie ${ }^{1}$, Samson W Fine ${ }^{1}$, Semra Olgac ${ }^{1}$ and Victor E Reuter ${ }^{1}$ \\ ${ }^{1}$ Department of Pathology, Memorial Sloan-Kettering Cancer Center, New York, NY, USA and ${ }^{2}$ Department of \\ Pathology, Weill Medical College of Cornell University, New York, NY, USA
}

\begin{abstract}
The presence of estrogen and progesterone-receptor-positive stroma is well known in renal mixed epithelial and stromal tumor, cystic nephroma, and angiomyolipoma with epithelial cysts. It has been suggested that the hormone receptor positivity in mixed epithelial and stromal tumor may be etiologically related to exogenous hormone intake-a phenomenon that has become more frequent in recent years. In the past few years, we have observed such stroma in some non-neoplastic kidneys, as well as in tumor-bearing kidneys away from the tumor. Herein we present our experience with 10 such cases. In a prospective manner, whenever we noted stroma resembling that in ovaries or müllerian organs (endometrial or cervical-like) in kidneys removed for any cause, immunohistochemical stains for estrogen and progesterone receptors were performed. There were eight males and two females among the group, with ages ranging from 11 months to 71 years. In six cases, the nephrectomies were performed for a non-functional kidney, and in three for tumors (one each of chromophobe, clear cell, and acquired cystic disease-associated renal cell carcinoma). One case was a partial nephrectomy for vesico-ureteric reflux, with upper pole hydronephrosis. Such stroma was present in nine cases as a non-mass forming proliferation around dilated, frequently inflamed pelvicalyceal system and collecting ducts. In one it was present at the periphery of an acquired cystic disease-associated renal cell carcinoma, as well as around non-tumorous cysts. The only common finding in all cases was a generalized or segmental hydronephrosis, or tumor compression-related focal obstruction. The stroma was positive for estrogen receptors in all 10 cases, and for progesterone receptors in seven. Thus, estrogen- and progesterone receptor-positive stroma can be present in the kidney, not only as a component of certain tumors, but also in association with non-neoplastic conditions. Its association with obstructive changes suggests that it may represent a metaplastic change in the renal interstitial cells surrounding these obstructed epithelial structures.
\end{abstract}

Modern Pathology (2008) 21, 60-65; doi:10.1038/modpathol.3800958; published online 14 September 2007

Keywords: estrogen- and progesterone receptor-positive stroma; ovarian and müllerian; non-neoplastic kidney; obstruction

The presence of stroma that variably has been described as ovarian, endometrial and müllerianlike, or paucicellular to hypercellular, is widely reported in renal tumors such as cystic nephroma, mixed epithelial-stromal tumors (MEST), and more recently in angiomyolipomas with epithelial cysts (AMLEC). ${ }^{1-7}$ In most of these tumors, this stroma

Correspondence: Dr SK Tickoo, MD, Department of Pathology, Memorial Sloan-Kettering Cancer Center, 1275 York Avenue, New York, NY 10021, USA.

E-mail: tickoos@mskcc.org

Received 06 February 2007; revised 23 July 2007; accepted 31 July 2007; published online 14 September 2007 also expresses estrogen and progesterone receptors (ER and PR). ER and PR positivity has also been described in otherwise usual angiomyolipomas, particularly those associated with tuberous sclerosis. ${ }^{8-10}$

To the best of our knowledge, outside of the settings of the above-mentioned tumors, ER- and PR-positive stroma in the kidneys has not been described. We initially noted such stromal proliferation in two kidneys resected for a non-functional status secondary to hydronephrosis. Immunohistochemical stains for ER and PR were strongly positive in this stroma in both the kidneys. Following this observation, we have been carefully analyzing all otherwise routinely sampled nephrectomy 
specimens resected for any primary pathology. Herein, we report our findings.

\section{Materials and methods}

After our initial observations on the two nephrectomy specimens mentioned previously, whenever we noted the presence of stroma that bore some resemblance to or created a mental image of the stroma in ovaries, endometrium, or cervix in nephrectomy specimens resected at our institutions for any disease (tumorous or non-tumorous) from the years 2003 to 2005, immunohistochemical stains for ER and PR were performed. Detailed morphologic analysis of the lesion, as well as the rest of the kidney was performed, and clinical details were obtained from the clinical records.

The location of such stroma was noted in each case, as were other gross and microscopic pathological findings. Immunohistochemical stainings were performed on one representative block containing such stroma, using antibodies against ER (clone 6F11; Ventana, Tucson, AZ, USA) and PR (clone 1E2; Ventana, Tucson, AZ, USA) performed on an automated slide stainer (Ventana BenchMark) according to the manufacturer's instructions, and as described previously. ${ }^{11}$ Any nuclear brown staining was considered a positive result. The percentage of positive-staining nuclei in the foci of stromal proliferation was calculated after counting as many nuclei as possible in the focus. Surrounding renal parenchyma, including the tubules, interstitium, and glomeruli, as well as the urothelium, were examined for any positive staining in each case.

One representative section from 10 other nephrectomy specimens without any stromal proliferation (from five male and five female patients) was also stained for both the receptors. Three of these were hydronephrotic kidneys.

\section{Results}

The clinicopathologic details of the 10 cases are presented in Table 1.

Interestingly, eight of the 10 cases were males. The ages ranged from 11 months to 71 years. To the best of our knowledge, none of the male patents had been on hormone therapy. One patient had received neoadjuvant chemotherapy for urothelial carcinoma of the bladder.

In nine of the kidneys, the stroma was present as a non-mass forming spindle cell proliferation around the dilated pelvicalyceal system, and intimately admixed with the collecting ducts close to the calyces (Figure 1a). In two of these nine kidneys, renal cell carcinoma extended into the renal medulla and compressed the calyceal system. Even in these cases, the spindle cell stromal proliferation was limited to the compressed calyces, and was not

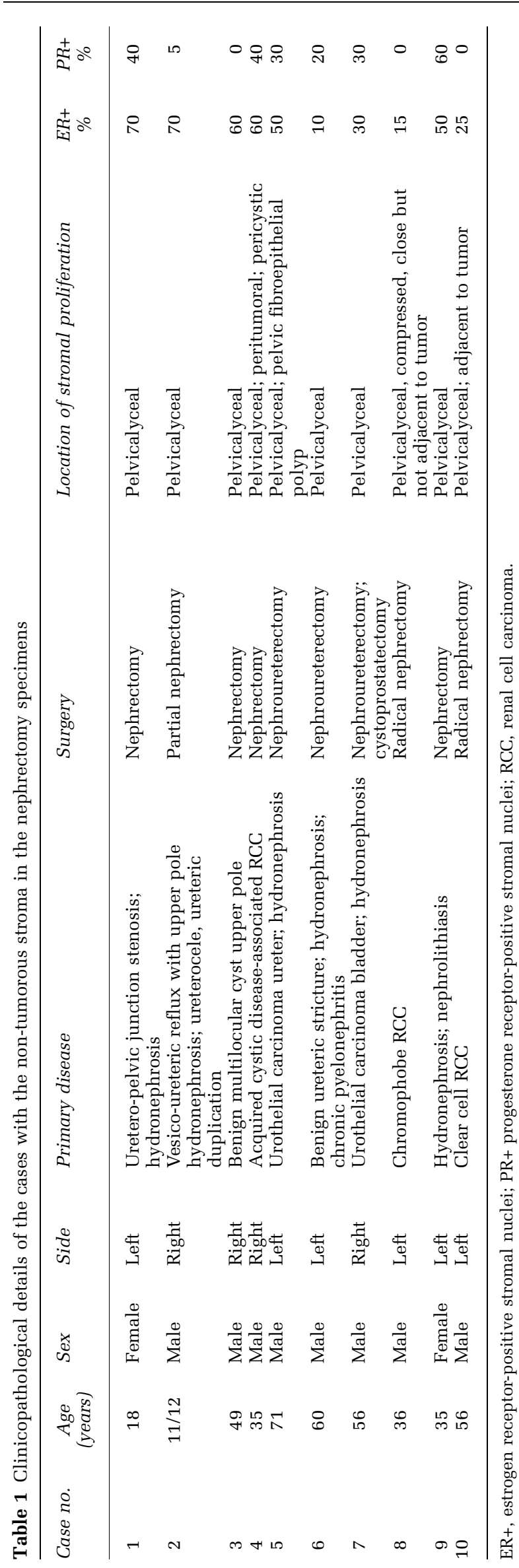



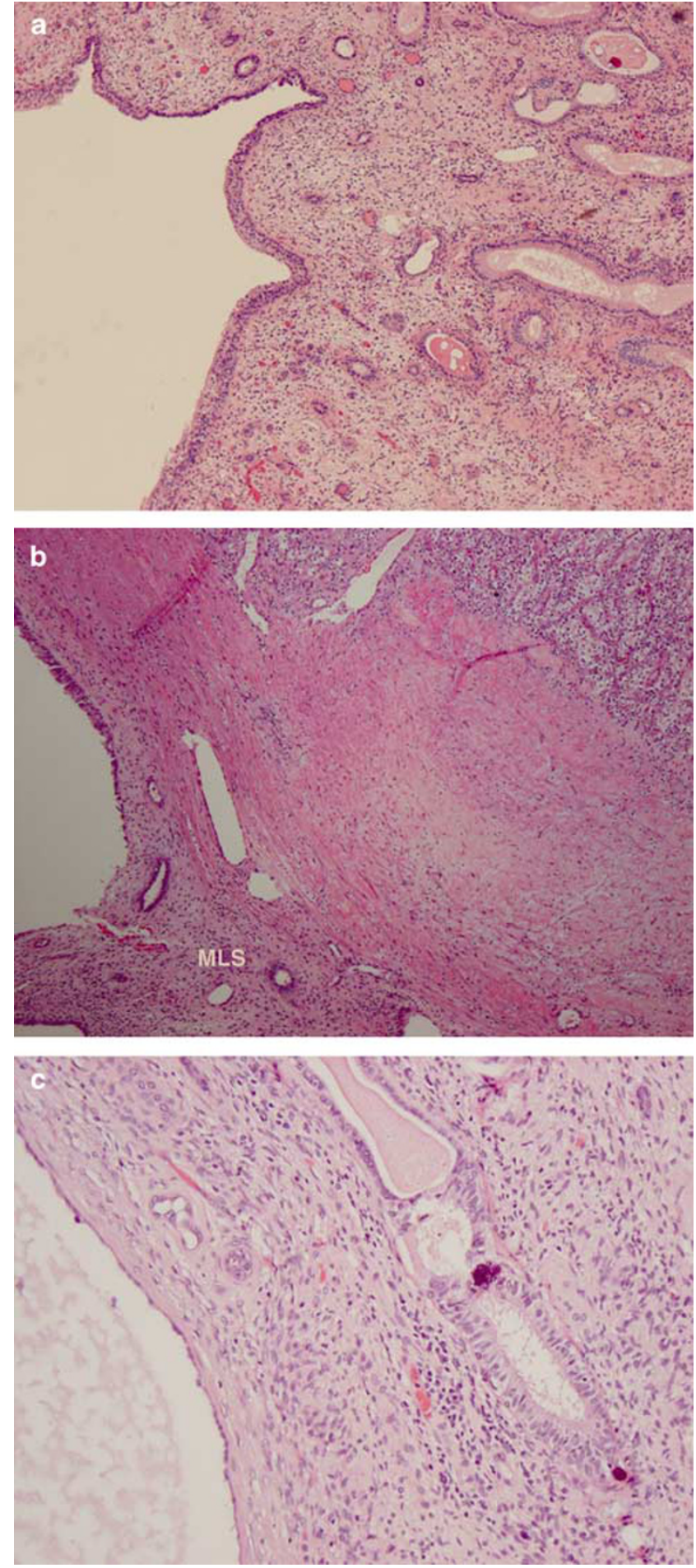

Figure 1 Non-tumorous stromal proliferation, (a) subjacent to calyceal urothelial lining and between dilated collecting ducts in a benign kidney with hydronephrosis, (b) underlying the urothelium (MLS), adjacent to a clear cell renal cell carcinoma extending close to the pelvicalyceal system, and (c) surrounding a benign cyst in a case of acquired cystic disease of the kidney.

present elsewhere adjacent, within, or distant to the tumor (Figure 1b). The outlines of the proliferation were never well defined, extending between the collecting ducts and merging imperceptibly with the more proximal renal interstitium. In the case of the end-stage kidney with acquired cystic disease, spindle cell hypercellularity was seen in the periphery of an acquired cystic disease-associated renal cell carcinoma (ACD-associated RCC).$^{12}$ In this case, similar but less cellular stroma was also observed around other non-neoplastic cysts within the kidney away from the tumor mass (Figure 1c). In five of the cases the spindle cell proliferation was associated with mild fibrosis. In two cases moderate, and another five minimal amount of inflammatory cells, predominantly lymphocytes, were admixed with the spindle cells. In general, the stromal proliferations ranged from short spindled to stellate cells arranged loosely (superficially resembling stroma of basal endometrium or endometrial polyps), to paucicellular stroma with plump nuclei and fibrotic background (superficially resembling endocervical stroma) to densely cellular stroma with wavy nuclei closely resembling ovarian-type stroma (Figure 2a-c). Specifically, the densely cellular, ovarian-type stroma was observed in only one case. No significant pleomorphism or mitotic activity was observed (rare mitoses were observed in two cases). The cytoplasm was pale-eosinophilic, and ranged from minimal to moderate in amount.

Immunohistochemical staining was positive for ER in all cases, whereas expression of PR was observed in seven (Table 1). Among the seven cases coexpressing ER and PR, ER positivity was more diffuse and stronger than PR in four (Figure 3a and b). Stains for Ki-67 revealed only minimally elevated (never more than 3\%) proliferation index compared with the surrounding interstitium. In two cases, PRbut not ER-positive nuclei were also observed in the renal parenchyma, away from the stromal lesions. These receptor-positive cells were located in some tubules, interstitial stromal cells, as well as the mesangial cells of glomeruli (Figure 3c). One of these cases was an 11-month-old male, and the other an 18-year-old female. Among the 10 control cases that did not show such stromal lesions, very occasional PR-positive renal interstitial cells were also observed in two cases; one a 37-year-old female and the other a male of 48 years. One of these two cases (the 48-year-old male) had hydronephrosis without any obvious stromal proliferation.

\section{Discussion}

Although human kidney is traditionally thought to be unresponsive to estrogen, some renal tumors like angiomyolipomas have been reported to increase in size in pregnancy ${ }^{13}$ or even secondary to oral contraceptive therapy. ${ }^{14}$ In animal studies, tumors that are closely related to human angiomyolipomas have also been reported to grow with estrogen therapy and regress on tamoxifen. ${ }^{15}$

In Hamster animal models, renal epithelial tumors have been shown to be inducible with estrogen. ${ }^{16,17}$ 

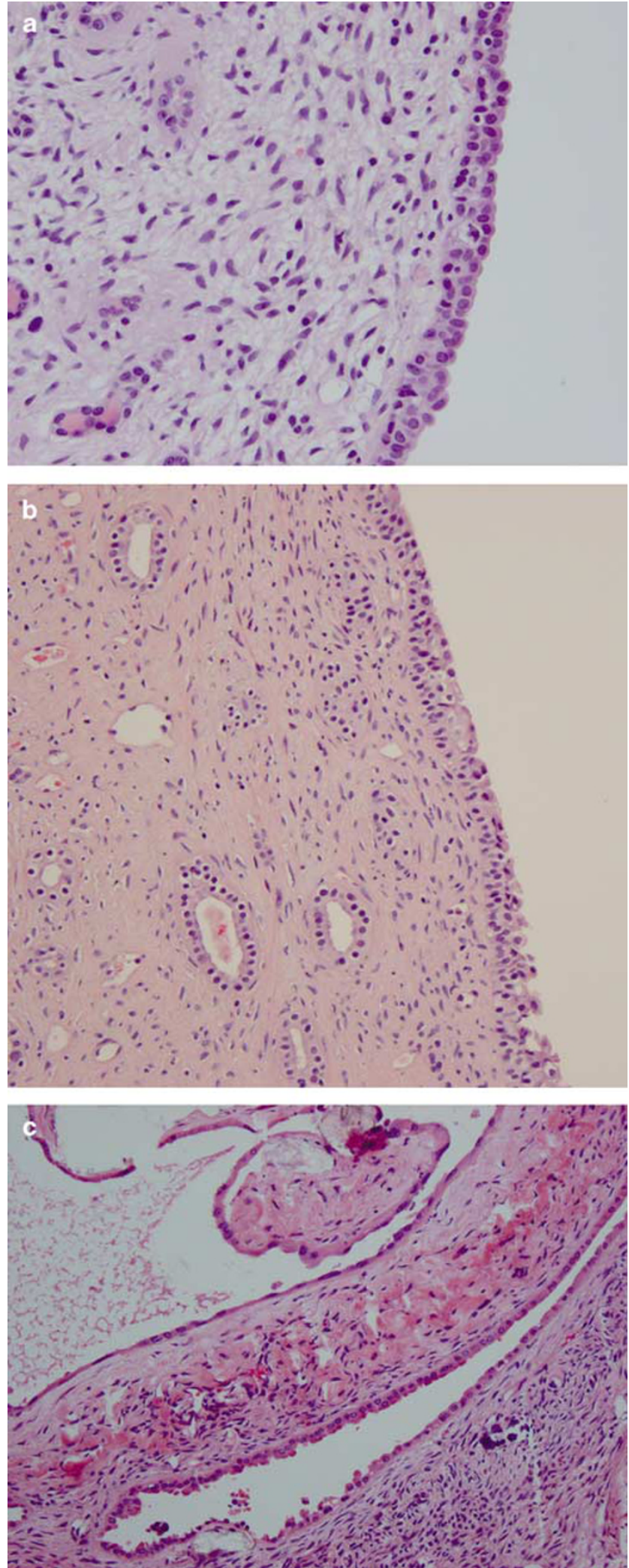

Figure 2 Higher magnification view of the stroma showing (a) widely spaced spindle to stellate cells with plump nuclei, (b) cells with more elongated nuclei in a fibrotic background, and (c) hypercellular stroma with wavy nuclei.
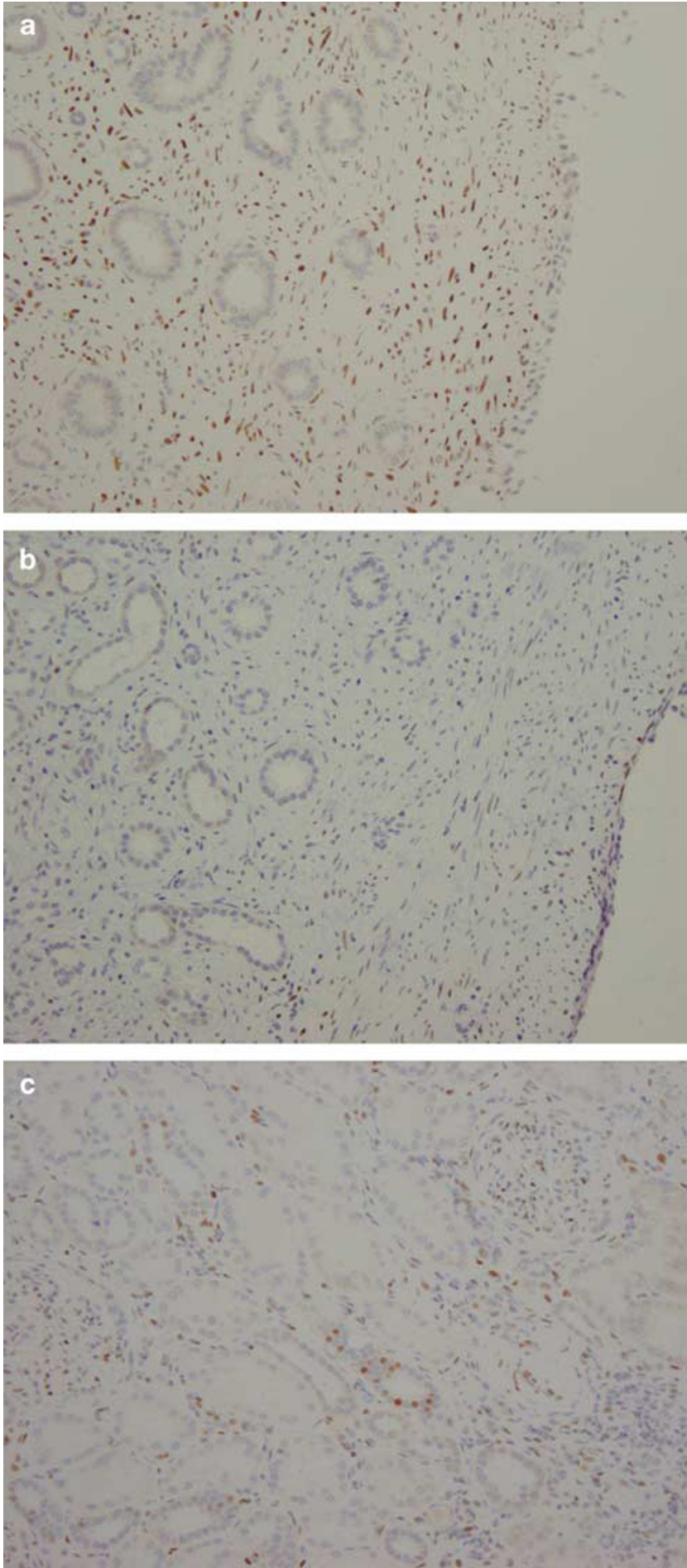

Figure 3 Immunohistochemical staining showing diffuse estrogen receptor positivity (a) and relatively focal progesterone receptor positivity (b) in non-tumorous stromal proliferation in one case. (c) Progesterone receptor positivity in tubular epithelium, interstitial cells, and glomerular mesangium in a case with non-tumorous stromal proliferation, away from the main lesion.

To the best of our knowledge, such initiation of renal carcinomas by estrogen in humans has not been reported. Nonetheless, expression of ER and PR in some human renal tumors has led to suggestions 
64

that some of these tumor types, particularly MEST, may be related to excessive exogenous estrogens.

Multiple reports indicate that rat/mouse kidneys show ER and PR expression, at least in the fetal stage. Such studies are quite rare in humans, but the presence of ER in human renal interstitial cells, both fetal and adult, have been reported. ${ }^{18-20}$ We observed $\mathrm{PR}$, but not ER, positivity in renal tubules, interstitial cells, and glomerular mesangium in two of the 10 cases with the stromal lesions under discussion. In the 10 kidneys without such lesions, very focal PR positivity was observed in the interstitial cells in only two cases. Therefore, it is reasonable to believe that cells potentially capable of being the source of the proliferation of ER/PR-positive stromal cells in human kidneys do exist, and these may be the progenitors of the ovarian-like/endometrioticlike/hypercellular stroma in some renal tumors, as well as in the non-tumorous settings as observed by us.

It is of interest that while most of the larger reported series on MEST and related lesions specifically mention the resemblance to ovarian-type stroma, each one of these in their microscopic descriptions, tables, or even accompanying figures also describe/show stroma that does not fit 'ovariantype' morphology. ${ }^{1-7}$ As one of the illustrated examples, the stroma in Figure 4 in the report by Turbiner et $a l^{6}$ is almost identical to that in our Figure 1a. Similarly, Mai et $a l^{5}$ observed endometriotic-like stroma in four of their 14 cases of MEST. Based on our personal experience with these tumors, we believe that while ovarian-like stroma is quite common, it does not constitute the exclusive type of stroma in most of these, and in many tumors no ovarian-like stroma is present at all. As a matter of fact, the stroma in many tumors, focally to predominantly or exclusively, resembles the stromal proliferations that we are describing in non-tumorous setting in nine of our 10 cases, and reminds one of the ER/PR-positive endometrial and endocervical stroma.

Renal tubular environment, conditioned by local tubular metabolism and function, is believed to control the local phenotype of interstitial fibroblasts, with resultant differential immunophenotypic expression of these interstitial cells in different anatomic zones of the kidney. ${ }^{21}$ Most of stromal proliferations were observed by us as non massforming foci either around dilated pelvicalyceal system and collecting ducts, or around cystic structures, and all of these were positive for ER and/or PR by immunohistochemical staining. The fact that these proliferations in all cases were observed only around cystically dilated pelvicalyceal structures and collecting ducts, cysts of acquired cystic disease of the kidney, and in the periphery of markedly cystic ACD-associated RCC, suggests that obstructive changes may have a primary pathogenetic role in such proliferations in the interstitial cells around these structures.
To the best of our knowledge, the localized proliferation of ER/PR-positive stroma in the kidneys not part of renal tumors like cystic nephroma, MEST, or AMLEC has not been described before. On review of the literature of obstructive nephropathies, we were not able to find any description of such ER/PR-positive stroma. ${ }^{22,23}$ However, we have noted stromal proliferation superficially resembling the one being described by us in illustrations on renal dysplasia and reflux nephropathy (figures 40-2 and 40-5) in a book chapter on developmental abnormalities of the kidney, but without any specific description of such stroma in the figure legends or the text. ${ }^{24}$ Interestingly, almost all the renal tumors with such stroma, as well as the lesions illustrated in the above-mentioned book chapter, have a prominent cystic component with the stroma usually surrounding these cystic structures. Our findings raise the possibility that the ER/PR stoma seen in association with renal tumors may also be a secondary (metaplastic) response to their cystic epithelial components and not necessarily a neoplastic constituent. The occurrence of the rarely reported sarcomatous transformation in MEST, including the occasional primary renal synovial sarcomas, ${ }^{25,26}$ does not completely exclude such a pathogenetic possibility, and can be explained as a subsequent malignant transformation in these lesions.

To conclude, ER/PR-positive stromal proliferation can be present in the kidney, not only as a part of MEST, cystic nephroma, and AMLEC, but also in association with non-neoplastic and other neoplastic conditions. Its association with renal obstructive changes raises the possibility that this may represent reactive and metaplastic changes in the renal interstitial cells surrounding these obstructed epithelial structures.

\section{References}

1 Antic T, Perry KT, Harrison K, et al. Mixed epithelial and stromal tumor of the kidney and cystic nephroma share overlapping features: reappraisal of 15 lesions. Archives Pathol Lab Med 2006;130:80-85.

2 Adsay NV, Eble JN, Srigley JR, et al. Mixed epithelial and stromal tumor of the kidney. Am J Surg Pathol 2000;24:958-970.

3 Michal M, Hes O, Bisceglia M, et al. Mixed epithelial and stromal tumors of the kidney. A report of 22 cases. Virchows Arch 2004;445:359-367.

4 Fine SW, Reuter VE, Epstein JI, et al. Angiomyolipoma with epithelial cysts (AMLEC): a distinct cystic variant of angiomyolipoma. Am J Surg Pathol 2006;30: 593-599.

5 Mai KT, Elkeilani A, Veinot JP. Mixed epithelial and stromal tumour (MEST) of the kidney: report of 14 cases with male and PEComatous variants and proposed histopathogenesis. Pathology 2007;39:235-240.

6 Turbiner J, Amin MB, Humphrey PA, et al. Cystic nephroma and mixed epithelial and stromal tumor of 
kidney: a detailed clinicopathologic analysis of 34 cases and proposal for renal epithelial and stromal tumor (REST) as a unifying term. Am J Surg Pathol 2007;31:489-500.

7 Steele R, Daroca Jr PJ, Hill S, et al. Multilocular renal cyst (cystic nephroma) with mullerian-like stroma. Urology 1994;43:549-553.

8 Henske EP, Ao X, Short MP, et al. Frequent progesterone receptor immunoreactivity in tuberous sclerosisassociated renal angiomyolipomas. Mod Pathol 1998;11: $665-668$.

9 Logginidou H, Ao X, Russo I, et al. Frequent estrogen and progesterone receptor immunoreactivity in renal angiomyolipomas from women with pulmonary lymphangioleiomyomatosis. Chest 2000;117:25-30.

10 L'Hostis H, Deminiere C, Ferriere JM, et al. Renal angiomyolipoma: a clinicopathologic, immunohistochemical, and follow-up study of 46 cases. Am J Surg Pathol 1999;23:1011-1020.

11 Darvishian F, Hummer AJ, Thaler HT, et al. Serous endometrial cancers that mimic endometrioid adenocarcinomas: a clinicopathologic and immunohistochemical study of a group of problematic cases. Am J Surg Pathol 2004;28:1568-1578.

12 Tickoo SK, dePeralta-Venturina MN, Harik LR, et al. Spectrum of epithelial neoplasms in end-stage renal disease: an experience from 66 tumor-bearing kidneys with emphasis on histologic patterns distinct from those in sporadic adult renal neoplasia. Am J Surg Pathol 2006;30:141-153.

13 Eble JN. Angiomyolipoma of kidney. Semin Diagn Pathol 1998;15:21-40.

14 Gould Rothberg BE, Grooms MC, Dharnidharka VR. Rapid growth of a kidney angiomyolipoma after initiation of oral contraceptive therapy. Obstet Gynecol 2006;108:734-736.

15 El-Hashemite N, Walker V, Kwiatkowski DJ. Estrogen enhances whereas tamoxifen retards development of Tsc mouse liver hemangioma: a tumor related to renal angiomyolipoma and pulmonary lymphangioleiomyomatosis. Cancer Res 2005;65:2474-2481.
16 Bhat HK, Hacker HJ, Bannasch P, et al. Localization of estrogen receptors in interstitial cells of hamster kidney and in estradiol-induced renal tumors as evidence of the mesenchymal origin of this neoplasm. Cancer Res 1993;53:5447-5451.

$17 \mathrm{Li} \mathrm{JJ,} \mathrm{Weroha} \mathrm{SJ,} \mathrm{Davis} \mathrm{MF,} \mathrm{et} \mathrm{al.} \mathrm{ER} \mathrm{and} \mathrm{PR} \mathrm{in}$ renomedullary interstitial cells during Syrian hamster estrogen-induced tumorigenesis: evidence for receptor-mediated oncogenesis. Endocrinology 2001;142: 4006-4014.

18 Brandenberger AW, Tee MK, Lee JY, et al. Tissue distribution of estrogen receptors alpha (ER-alpha) and beta (ER-beta) mRNA in the midgestational human fetus. J Clin Endocrinol Metab 1997;82:3509-3512.

19 Kuiper GG, Carlsson B, Grandien K, et al. Comparison of the ligand binding specificity and transcript tissue distribution of estrogen receptors alpha and beta. Endocrinology 1997;138:863-870.

20 Taylor AH, Al-Azzawi F. Immunolocalisation of oestrogen receptor beta in human tissues. J Mol Endocrinol 2000;24:145-155.

21 Marxer-Meier A, Hegyi I, Loffing J, et al. Postnatal maturation of renal cortical peritubular fibroblasts in the rat. Anat Embryol 1998;197:143-153.

22 Klahr S, Morrissey J. Obstructive nephropathy and renal fibrosis. Am J Physiol 2002;283:F861-F875.

23 Becker A, Baum M. Obstructive uropathy. Early Hum Dev 2006;82:15-22.

24 Gilbert-Barness E, Lacson A. Developmental abnormalities of the kidney. In: Mills SE (ed). Sternberg's Diagnostic Surgical Pathology, 4th edn. Lippincott Williams and Wilkins: Philadelphia, 2004, pp 1845.

25 Adsay NV, Che M, Basturk O, et al. Sarcomatous transformation in mixed epithelial stromal tumors of the kidney (malignant MEST). Lab Invest 2004;84: 135A.

26 Argani P, Faria PA, Epstein JI, et al. Primary renal synovial sarcoma: molecular and morphologic delineation of an entity previously included among embryonal sarcomas of the kidney. Am J Surg Pathol 2000;24:1087-1096. 